\title{
INFLUÊNCIA DO MÓDULO DO SILICATO DE SÓDIO NA FLOTAÇÃO DE MINÉRIO DE FERRO: ESTUDOS FUNDAMENTAIS
}

Renato Souza Arantes '

Tamires Fonseca Souza '

Rosa Malena Fernandes Lima '

\section{Resumo}

Este artigo apresenta a influência do módulo do silicato de sódio sobre as flotabilidades e cargas superficiais da hematita e do quartzo com oleato de sódio. Baseado nos resultados dos ensaios de microflotação, efetuados em $\mathrm{pH}$ 7, foi observado que as flotabilidades da hematita foram maiores do que as flotabilidades do quartzo para todas as concentrações de silicato de sódio testadas independentemente do módulo do reagente. Os valores de potencial zeta da hematita tornaram-se negativos para valores de $\mathrm{pH}$ menores do que o ponto isoelétrico do mineral $(\mathrm{pH} 7,2)$ e cargas negativas maiores para valores de $\mathrm{pH}$ acima do seu ponto isoelétrico, o que sugere a precipitação de $\mathrm{SiO}_{2 \text { amorfa }}$ e adsorção de espécies aniônicas presentes na solução sobre a superfície do mineral, especialmente para valores de pH acima de 10. No caso do quartzo, não foi verificada influência significativa do módulo do reagente tanto nas flotabilidades quanto nos valores de potencial zeta.

Palavras-chave: Hematita; Quartzo; Flotação; Silicato de sódio.

\section{INFLUENCE OF SODIUM SILICATE MODULUS ON IRON ORE FLOTATION: FUNDAMENTAL STUDIES}

\begin{abstract}
The influence of sodium silicate modulus on the floatabilities and surface charges of hematite and quartz with sodium oleate is presented herein. Based on microflotation test results, performed at $\mathrm{pH} 7$, it was observed that the floatabilities of hematite were higher than the floatabilities of quartz for all concentrations of sodium silicate tested, independent of reagent modus. The values of zeta potential of hematite become negative for $\mathrm{pH}$ values smaller than the mineral isoelectric point (7.2) and have bigger negative charges for $\mathrm{pH}$ values above its isolectric point, which suggests precipitation of $\mathrm{SiO}_{2 \text { amorphous }}$ and adsorption of anionic specimens present in solution on the mineral surface, especially for values of $\mathrm{pH}$ above 10 . In the case of quartz, we were unable to verify a significant influence of the reagent modulus on floatabilities and values of the zeta potential.
\end{abstract}

Keywords: Hematite; Quartz; Flotation; Sodium silicate.

\section{INTRODUÇÃO}

A escolha da rota de beneficiamento para um dado minério é efetuada em função dos aspectos mineralógicos, distribuição granulométrica e teor. No caso específico de minério de ferro, a flotação catiônica reversa, utilizando amina como coletor de silicatos (quartzo e outros) e amido como depressor dos minerais de ferro, é largamente utilizada para concentração da fração - $105 \mu \mathrm{m}$ de minérios do Quadrilátero Ferrífero. No entanto, para minérios cada vez mais pobres (teores entre 25 a $45 \%$ ) e para o reprocessamento de rejeitos depositados em barragens, bem como para o aproveitamento de lamas descartadas nas etapas de deslamagem, pode-se pensar em empregar a flotação aniônica, utilizando sabões de ácidos graxos como coletor dos minerais de ferro $[1,2]$.

O silicato de sódio $\left(\mathrm{mNaO}_{2}: \mathrm{nSiO}_{2} \mathrm{O}\right)$, usualmente é utilizado como dispersante no beneficiamento a úmido de minérios de ferro [3,4]. Na flotação de rocha fosfática, dependendo do $\mathrm{pH}$, concentração e módulo do reagente ( razão $n / m$ ), o mesmo é empregado como depressor de silicatos e carbonatos [5]. As equações de equilíbrio entre

'Programa de Pós-graduação em Engenharia Mineral - PPGEM, Departamento de Engenharia de Minas - DEMIN, Universidade Federal de Ouro Preto - UFOP, Campus Morro do Cruzeiro, Ouro Preto, MG, Brasil. E-mail: rosa@demin.ufop.br 
as espécies de silicato de sódio presentes em meio aquoso, segundo modelo de Marinakis e Shergold [6], modificado por Qi et al. [7] são:

$\mathrm{SiO}_{2(\text { s, amorfo })}+2 \mathrm{H}_{2} \mathrm{O}=\mathrm{Si}(\mathrm{OH})_{4} \log \mathrm{K}=-2,7$

$\mathrm{Si}(\mathrm{OH})_{4}=\mathrm{SiO}(\mathrm{OH})_{3}{ }^{-}+\mathrm{H}^{+} \log \mathrm{K}=-9,46$

$\mathrm{SiO}(\mathrm{OH})_{3}{ }^{-}=\mathrm{SiO}_{2}(\mathrm{OH})_{2}{ }^{2-}+\mathrm{H}^{+} \log \mathrm{K}=-12,56$

$4 \mathrm{Si}(\mathrm{OH})_{4}=\mathrm{SiO}_{4}(\mathrm{OH})_{6}{ }^{2-}+2 \mathrm{H}^{+}+4 \mathrm{H}_{2} \mathrm{O} \log \mathrm{K}=-12,57$

Rao et al. [8] efetuaram estudos de flotação direta $(\mathrm{pH} 9-10,5)$ de uma lama de minério de ferro $(54.55 \%$ $\mathrm{Fe}, 6,72 \% \mathrm{SiO}_{2}$ e 6,80\% $\mathrm{Al}_{2} \mathrm{O}_{3}$ ), usando silicato de sódio como depressor, oleato de sódio como coletor e polpa com $40 \%$ de sólidos. $\mathrm{Na}$ condição otimizada $(0,2 \mathrm{~kg} / \mathrm{t}$ de silicato de sódio de módulo 2,19 e $0,5 \mathrm{~kg} / \mathrm{t}$ oleato de sódio) foi obtido concentrado com $58,89 \% \mathrm{Fe}, 4,68 \% \mathrm{SiO}_{2}$, e $5,28 \%$ $\mathrm{Al}_{2} \mathrm{O}_{3}$ com recuperação mássica de $38,74 \%$ e recuperação metalúrgica de 4I, I 3\%. Nesse estudo verificou-se que tanto a concentração de silicato de sódio quanto o módulo do reagente tiveram efeito significativo no processo.

Arantes e Lima [9], usando planejamento estatístico de experimentos a dois níveis, estudaram a influência da dosagem de oleato de sódio (300 e $1200 \mathrm{~g} / \mathrm{t}$ ), \% de sólidos (60 e 70\%), módulo ( I; 2,26 e 3,33) e dosagem de silicato de sódio (25 e $500 \mathrm{~g} / \mathrm{t}$ ) na flotação de uma amostra de minério de ferro $\left(42,8 \% \mathrm{Fe}\right.$ e $\left.37,5 \% \mathrm{SiO}_{2}\right)$ em $\mathrm{pH} 7$, previamente determinado por Lopes e Lima [10]. Nesses estudos, verificou-se que a recuperação metalúrgica foi acima de $90 \%$ com teores de $55 \%$ Fe. Os maiores teores de Fe e menores teores de $\mathrm{SiO}_{2}$ nos concentrados foram obtidos para polpa com $60 \%$ de sólidos, $25 \mathrm{~g} / \mathrm{t}$ de silicato de sódio (módulo = 3,33) e l $200 \mathrm{~g} / \mathrm{t}$ de oleato de sódio. $\mathrm{O}$ aumento do módulo do silicato de sódio de I para 3,33 acarretou aumento tanto na recuperação ( $1,2 \%)$ quanto no teor $(2,3 \%)$ de Fe e decrescimento do teor de $\mathrm{SiO}_{2}(2,3 \%)$ nos concentrados obtidos. Visando ao entendimento dos resultados de flotação em bancada dos ensaios efetuados por Arantes e Lima [9], esse trabalho apresenta o efeito do módulo do silicato de sódio sobre a flotabilidade e carga superficial da hematita e quartzo com oleato de sódio em $\mathrm{pH} 7$.

\section{MATERIAS E MÉTODOS}

\section{I Amostras Minerais e Reagentes}

A composição química e perda por calcinação das amostras minerais (hematita e quartzo), os reagentes usados nos ensaios de microflotação e medidas de potencial zeta estão apresentadas nas Tabelas I e 2, respectivamente.

A preparação das soluções primárias $(5 \% \mathrm{p} / \mathrm{v})$ de silicato de sódio de diferentes módulos constou da pesagem dos reagentes, descontando a água presente nos mesmos. A saponificação do ácido oleico com $\mathrm{NaOH}(10 \% \mathrm{p} / \mathrm{v})$ e posterior diluição para concentração a $1 \%$ p/v foi efetuada conforme metodologia descrita por Arantes e Lima [9].

\subsection{Testes de Microflotação e Medidas de Potencial Zeta}

Os ensaios de microflotação, em função da dosagem e módulo do silicato de sódio (Tabela 3), foram efetuados em triplicata nas condições de máxima flotabilidade da hematita $(\mathrm{pH}=7$ e $50 \mathrm{mg} / \mathrm{L}$ de oleato de sódio), determinadas previamente por Lopes e Lima [10]. As amostras minerais de I g (faixa granulométrica - $106+43 \mu \mathrm{m}$ ) eram introduzidas

Tabela I. Composição química e perda por calcinação das amostras de hematita e quartzo, usadas nos ensaios de microflotação e medidas de potencial zeta

\begin{tabular}{|c|c|c|c|c|}
\hline \multirow{3}{*}{$\begin{array}{c}\text { Teores } \\
\text { (\%) }\end{array}$} & \multicolumn{4}{|c|}{ Minerais } \\
\hline & \multicolumn{2}{|c|}{ Hematita } & \multicolumn{2}{|c|}{ Quartzo } \\
\hline & Microflotação & Potential zeta & Microflotação & Potential zeta \\
\hline $\mathrm{Fe}_{\text {Total }}$ & 68,89 & 69,30 & - & - \\
\hline $\mathrm{FeO}$ & 0,37 & 0,35 & - & - \\
\hline $\mathrm{Fe}_{2} \mathrm{O}_{3}$ & 98,08 & 98,7 & 0,05 & 0,34 \\
\hline $\mathrm{SiO}_{2}$ & 0,27 & 0,30 & 99,00 & 98,50 \\
\hline $\mathrm{Al}_{2} \mathrm{O}_{3}$ & 0,17 & 0,29 & 0,22 & 0,41 \\
\hline $\mathrm{P}$ & 0,02 & 0,02 & - & - \\
\hline$M n$ & 0,073 & 0,053 & - & - \\
\hline $\mathrm{CaO}$ & 0,07 & 0,06 & 0,20 & 0,03 \\
\hline $\mathrm{MgO}$ & 0,03 & 0,03 & - & 0,03 \\
\hline $\mathrm{TiO}_{2}$ & 0,02 & 0,02 & 0,04 & 0,04 \\
\hline $\mathrm{Na}_{2} \mathrm{O}$ & - & - & 0,03 & 0,02 \\
\hline PPC & 0,35 & 0,38 & - & 0,06 \\
\hline
\end{tabular}

PPC - perda por calcinação. 
Tabela 2. Reagentes utilizados nos testes de microflotação e medidas de potencial zeta

\begin{tabular}{|c|c|c|c|c|c|c|}
\hline \multicolumn{7}{|c|}{ Depressor: Silicato de sódio (comercial) } \\
\hline \multirow{2}{*}{ Módulo } & \multicolumn{3}{|c|}{ Composição química } & \multicolumn{3}{|c|}{ Propriedades } \\
\hline & $\mathrm{SiO}_{2}$ & $\mathrm{Na}_{2} \mathrm{O}$ & $\mathrm{H}_{2} \mathrm{O}$ & \% de sólidos & Densidade (g/L) & Viscosidade (cP) \\
\hline I & 28,8 & 28,8 & 42,5 & 57,6 & - & - \\
\hline 2,26 & 33,3 & 14,7 & 52,0 & 48,0 & 1,6 & 1350 \\
\hline 3,33 & 29,9 & 8,9 & 61,3 & 38,7 & $\mathrm{I}, 4$ & 540 \\
\hline \multicolumn{7}{|c|}{ Coletor: Oleato de sódio - Concentração de 50 mg/L (Lopes e Lima) [10] } \\
\hline \multicolumn{7}{|c|}{ Modulador de pH: $\mathrm{NaOH}$ (Dinâmica) e $\mathrm{HCl}$ (Vetec) } \\
\hline \multicolumn{7}{|c|}{ Meio iônico: $\mathrm{NaCl}$ (Vetec) - Concentração de $10^{-3} \mathrm{~mol} / \mathrm{L}$} \\
\hline
\end{tabular}

Tabela 3. Concentração ( $\mathrm{mol} / \mathrm{L}$ ) de $\mathrm{SiO}_{2}$ em função da dosagem de silicato de sódio

\begin{tabular}{|c|c|c|c|c|c|c|}
\hline \multicolumn{2}{|c|}{ Silicato de sódio (SS) } & \multicolumn{5}{|c|}{ Dosagem de SS (mg/L) } \\
\hline Módulo & Notação & $\mathbf{I}$ & 10 & 20 & 50 & 100 \\
\hline I & SSI & $8,32 \times 10^{-6}$ & $8,32 \times 10^{-5}$ & $1,66 \times 10^{-4}$ & $4,16 \times 10^{-4}$ & $8,32 \times 10^{-4}$ \\
\hline 2,26 & SS2,26 & $1,15 \times 10^{-5}$ & $1,15 \times 10^{-4}$ & $2,31 \times 10^{-4}$ & $5,77 \times 10^{-4}$ & $1,15 \times 10^{-3}$ \\
\hline 3,33 & SS3,33 & $1,28 \times 10^{-5}$ & $1,28 \times 10^{-4}$ & $2,56 \times 10^{-4}$ & $6,4 \times 10^{-4}$ & $1,29 \times 10^{-3}$ \\
\hline
\end{tabular}

no tubo de Hallimond modificado, adicionava-se $100 \mathrm{~mL}$ da solução de silicato de sódio na dosagem adequada em $\mathrm{pH}$ previamente ajustado para 7 e efetuava-se 0 condicionamento por 6 min. Em seguida, era introduzido o oleato de sódio, ajustava novamente o $\mathrm{pH}$ para 7, caso necessário, e procedia-se o condicionamento de 4 e 6 min., respectivamente para a hematita e para o quartzo. Então, o volume da célula $(250 \mathrm{~mL})$ era completado com água destilada em $\mathrm{pH}$ 7. Finalmente, as amostras minerais eram flotadas por um minuto, utilizando vazão de $60 \mathrm{~mL} / \mathrm{min}$ de nitrogênio comercial.

Para determinação do potencial zeta com réplica das amostras minerais $-10 \mu \mathrm{m}$ (suspensão de $0,01 \%$ ) foi utilizado o zetâmetro Malvern Zetasizer Nano Z - ZEN 2600 do Laboratório de Propriedades Interfaciais do DEMIN/UFOP. Foram levantadas curvas de potencial zeta dos minerais na ausência e condicionados com silicato de sódio na concentração de $100 \mathrm{mg} / \mathrm{L}$. As concentrações correspondentes de $\mathrm{SiO}_{2}$ (mol/L) dos silicatos de sódio de diferentes módulos podem ser vistas na Tabela 3 . Como meio iônico foi utilizada $\mathrm{NaCl}$ na concentração de $10^{-3} \mathrm{~mol} / \mathrm{L}$ em todos os ensaios efetuados.

\section{RESULTADOS E DISCUSSÃO}

\section{I Espécies de Silicato em Meio Aquoso}

Na Figura I está apresentado o digrama de espécies de silicato de sódio em meio aquoso em função do $\mathrm{pH}$, que foi calculado, usando as constantes de equilíbrio das Equações I a 4 para concentração de $100 \mathrm{mg} / \mathrm{L}$ de silicato de sódio de módulo $3,33\left(1,29 \times 10^{-3} \mathrm{~mol} / \mathrm{L}\right.$ de $\left.\mathrm{SiO}_{2}\right)$. De modo geral, pode-se afirmar que a concentração das espécies de silicato presentes em meio aquoso depende da concentração do reagente adicionado (Tabela 3) e do pH.

Observa-se pela Figura I, que em meio aquoso em $\mathrm{pH} 7$ a espécie predominante é o $\mathrm{SiO}_{\text {2amorfa }}$, da ordem de $10^{-3} \mathrm{mg}$. As concentrações de $\mathrm{Si}(\mathrm{OH})_{4}$ e $\mathrm{SiO}(\mathrm{OH})_{3}{ }^{-}$são da ordem de $10^{-5} \mathrm{~mol} / \mathrm{L}$ e $10^{-8} \mathrm{~mol} / \mathrm{L}$, respectivamente.

\subsection{Microflotação e Potencial Zeta}

Observa-se pela Figura 2 que as flotabilidades da hematita em pH 7 decresceram de $20 \%$ em relação à flotabilidade máxima (100\%) do mineral com oleato de sódio [10] para as concentrações de SS3,33 de 50 e $100 \mathrm{mg} / \mathrm{L}$, o que pode estar relacionado com as baixas concentrações das espécies aniônicas dos reagentes SSI e SS2,26 neste valor de $\mathrm{pH}$. No caso do quartzo (Figura 3) para dosagens maiores o decréscimo da flotabilidade foi da mesma ordem de grandeza da hematita. No entanto, não foi observada diferença significativa nos resultados obtidos com os reagentes de diferentes módulos. Estes resultados explicam a alta recuperação de $\mathrm{Fe}$ e altos teores de $\mathrm{SiO}_{2}$ nos concentrados obtidos por Arantes e Lima [9] e Lopes e Lima [10].

Observa-se pela Figura 4, que os valores de potencial zeta da hematita na ausência de reagente $\left(\mathrm{NaCl}=10^{-3} \mathrm{M}\right)$ caiu bruscamente de $13 \mathrm{mV}$ em pH 6,8 para $-32 \mathrm{mV}$ em pH 7,9.

Pela curva $\mathrm{NaCl}=1 \mathrm{mM}$, o ponto isoelétrico da hematita ocorreu em pH 7,2 [I I]. Os valores de potencial zeta da hematita após condicionamento com SSI foi negativo em toda faixa de $\mathrm{pH}$. Para valores de $\mathrm{pH}$ abaixo de 7 , a espécie predominante em meio aquoso é o $\mathrm{SiO}_{2(\text { s,amorfa) }}$. Para valores de $\mathrm{pH}$ acima de 7, verifica-se $o$ aumento das espécies aniônicas (Figura I), que se adsorveram sobre a superfície do mineral. Os valores de potencial zeta da hematita condicionada com SS2,26 e SS3,33, foram praticamente iguais, o que está relacionado com concentrações de espécies de 
$\mathrm{SiO}_{2(\text { s.amorfa) }}$ e aniônicas da mesma ordem de grandeza para os dois reagentes. Comportamento similar foi observado nos valores de potencial zeta da magnetita condicionada com silicato de sódio $\left(\mathrm{Na}_{2} \mathrm{OSiO}_{3} .9 \mathrm{H}_{2} \mathrm{O}\right)$ [12]. Baseado no modelo de complexação superficial para quantificar a borda de adsorção de silicatos sobre óxidos de ferro (goethita, hematita e magnetita) Jordean et al. [13] concluíram que dois tipos de complexos superficiais $\left(\equiv \mathrm{FeH}_{3} \mathrm{SiO}_{4} \mathrm{e} \equiv \mathrm{FeH}_{2} \mathrm{SiO}_{4}^{-}\right)$se

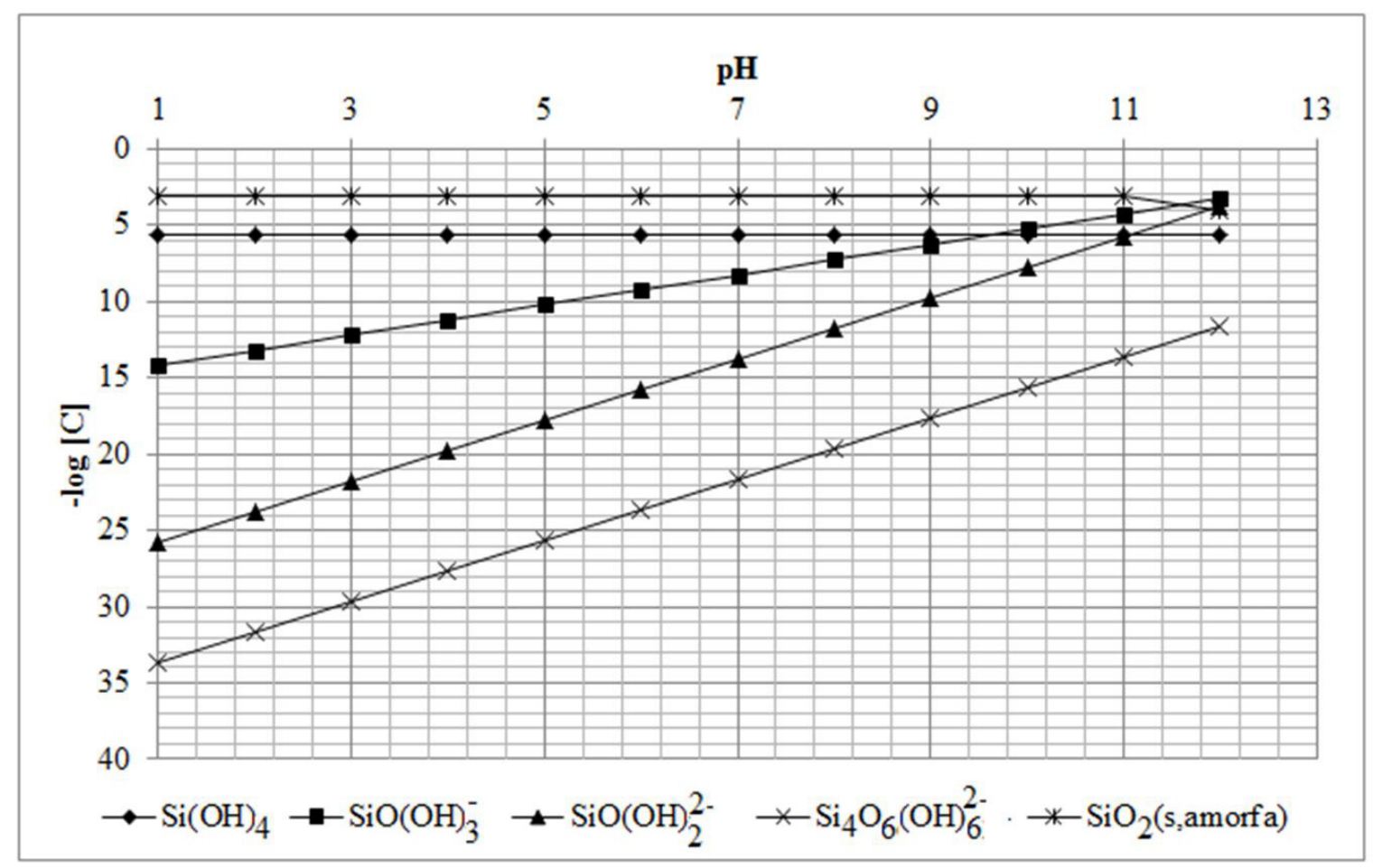

Figura I. Espécies de silicato em meio aquoso $(\mathrm{SS} 3,33=100 \mathrm{mg} / \mathrm{L})$ em função do $\mathrm{pH}$.

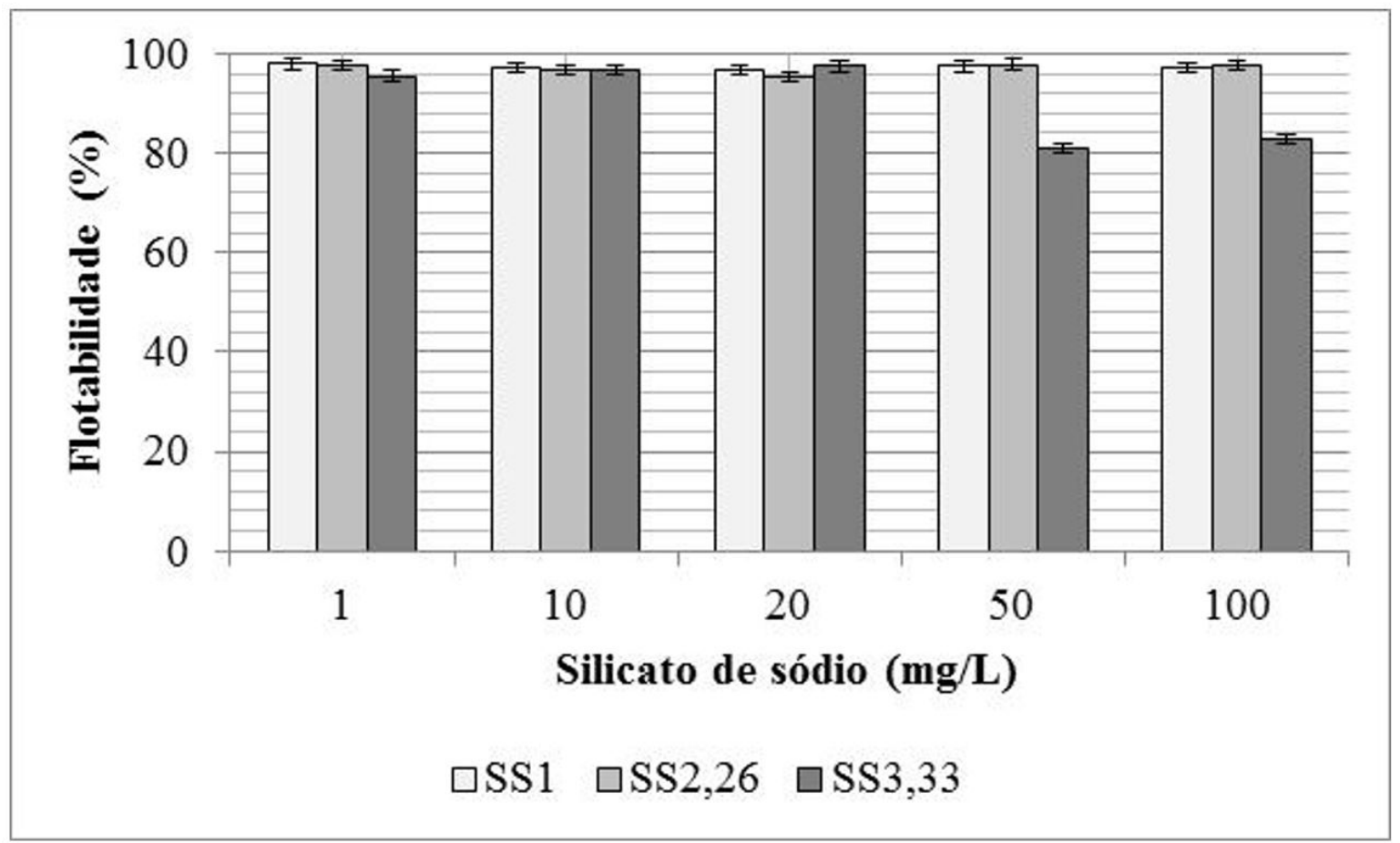

Figura 2. Flotabilidade da hematita em função da dosagem e módulo de silicato de sódio em pH 7 e oleato de sódio (50mg/L). 


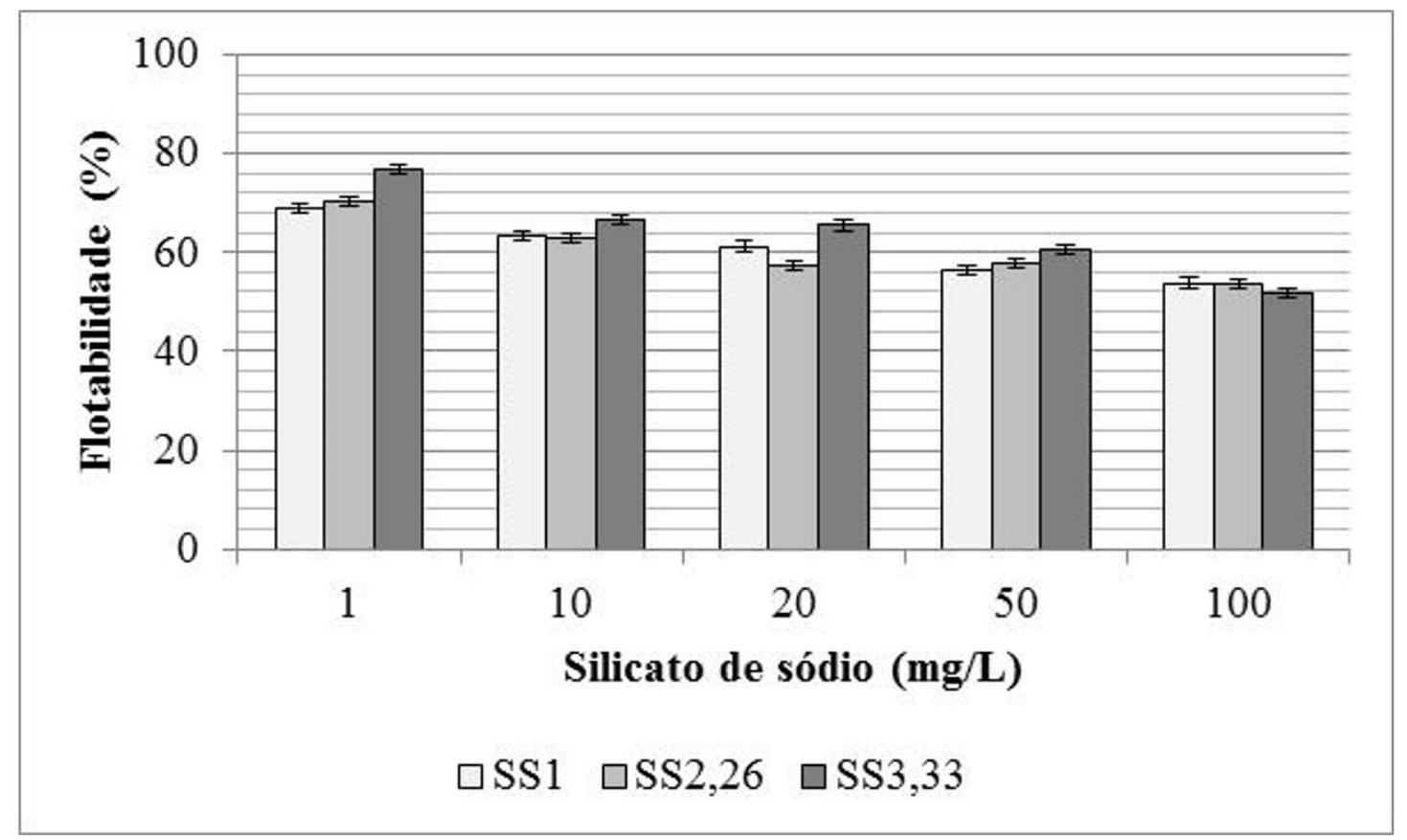

Figura 3. Flotabilidade do quartzo em função da dosagem e módulo de silicato de sódio em pH 7 e 50 mg/L de oleato de sódio.

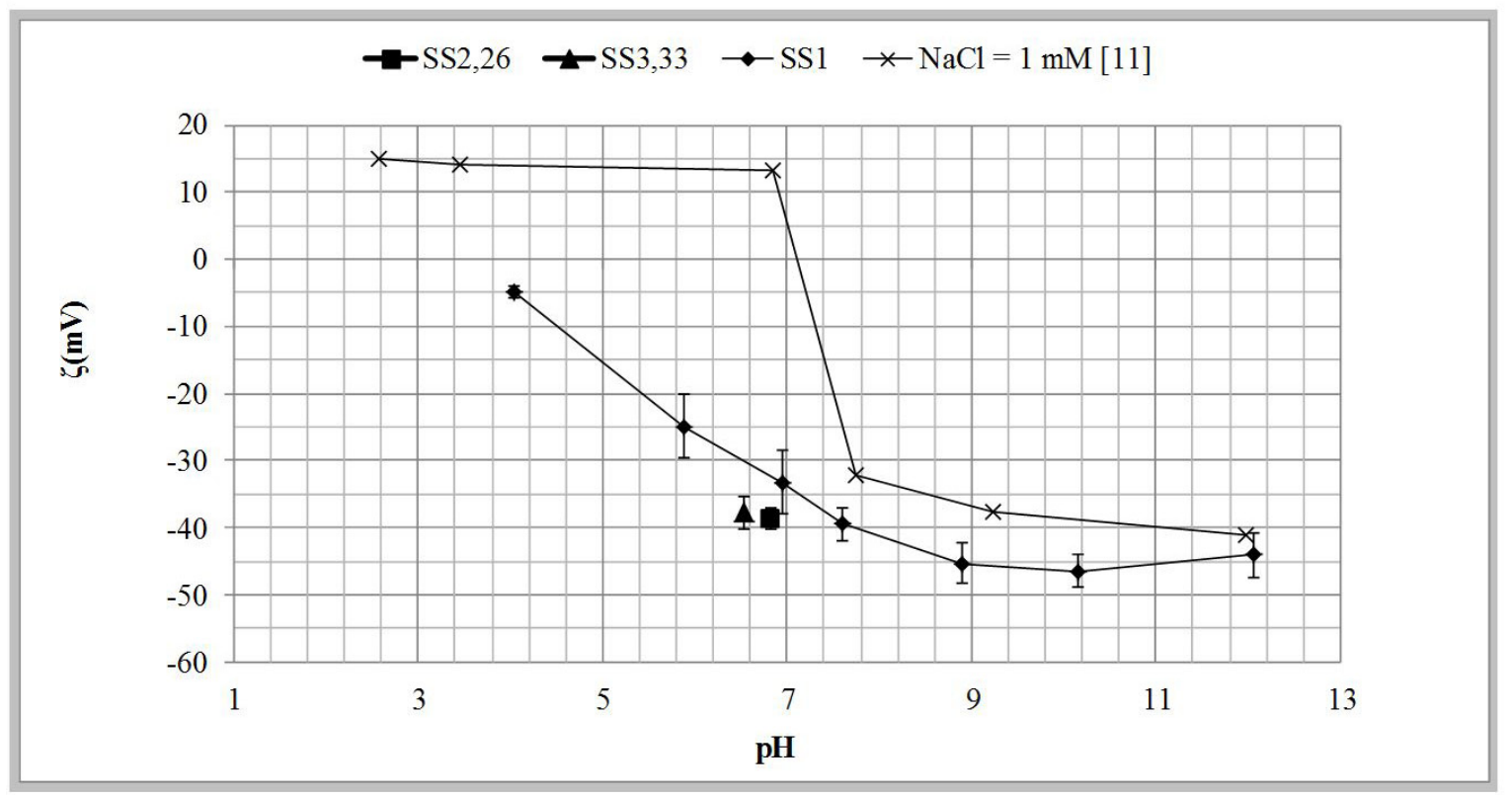

Figura 4. Potencial zeta da hematita na ausência e presença de silicato de sódio ( $100 \mathrm{mg} / \mathrm{L})$.

formam nas superfícies dos mesmos. Jolstera et al. [14] em estudos de adsorção de espécies de silicato solúveis $\left(\mathrm{Si}(\mathrm{OH})_{4}\right)$ sobre a maghemita $\left(\gamma-\mathrm{Fe}_{2} \mathrm{O}_{3}\right)$ por titulação potenciométrica de alta precisão, análises espectroscópicas e determinações de potencial zeta sugeriram três complexos superficiais $\left(\equiv \mathrm{FeOSiO}_{2}(\mathrm{OH})_{3} ;=\mathrm{FeOSiO}(\mathrm{OH})_{2}{ }^{-}\right.$e $\left.\equiv \mathrm{FeOSiO}_{2}(\mathrm{OH})^{2-}\right)$ sobre a superfície da maghemita.
Pela Figura 5 , observa-se que os valores de potencial zeta do quartzo condicionado com silicato de sódio de diferentes módulos não variaram significativamente e foram praticamente iguais aos valores de potencial zeta do mineral na ausência de silicato de sódio. Estes resultados estão coerentes com os resultados dos ensaios de microflotação (Figura 3), uma vez que em pH 7 a espécie predominante é o $\mathrm{SiO}_{2(\text { s,amorfa) }}$. 


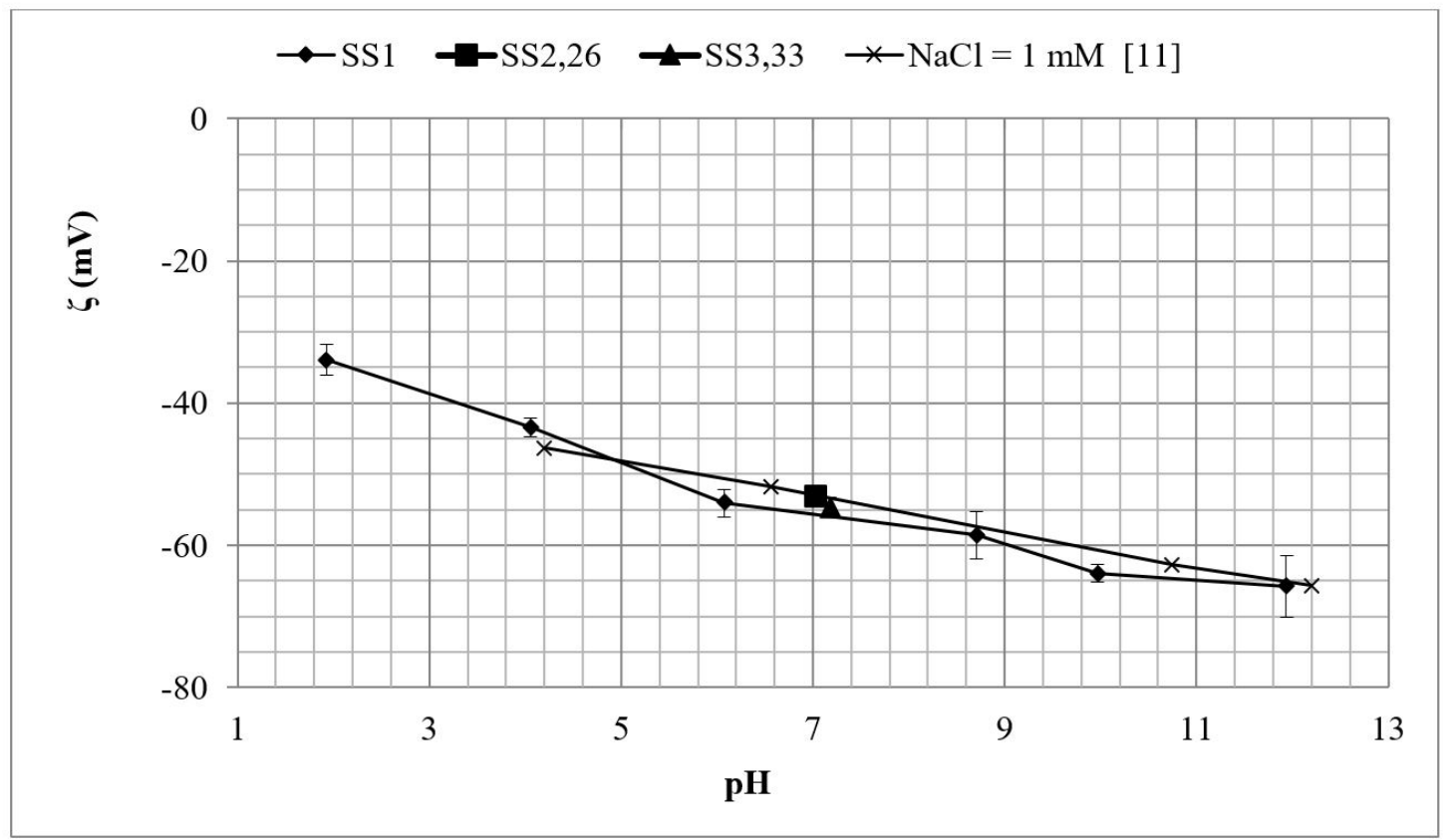

Figura 5. Potencial zeta do quartzo na ausência e presença de silicato de sódio ( $100 \mathrm{mg} / \mathrm{L})$.

\section{CONCLUSÕES}

Baseado nos resultados dos ensaios de microflotação foi observado que as flotabilidades da hematita foram maiores do que as flotabilidades do quartzo para todas as concentrações de silicato de sódio testadas independentemente do módulo do reagente. Os valores de potencial zeta da hematita tornaram-se negativos para valores de $\mathrm{pH}$ abaixo do ponto isoelétrico do mineral ( $\mathrm{pH} 7,2)$ e cargas negativas maiores para valores de $\mathrm{pH}$ acima do ponto isoelétrico, o que sugere a precipitação de $\mathrm{SiO}_{2(\text { s,amorfa })}$ e adsorção de espécies aniônicas presentes na solução, especialmente para valores de $\mathrm{pH}$ acima de 10 . No caso do quartzo, não foi verificada influência significativa do módulo do reagente tanto nas flotabilidades quanto nos valores de potencial zeta.

\section{Agradecimentos}

Os autores agradecem as bolsas concedidas pelo CNPq e pela FAPEMIG e aos auxílios financeiros concedidos pelo CNPq, Vale e CAPES.

\section{REFERÊNCIAS}

I Houot R. Beneficiation of iron ore by flotation - review of industrial and potential applications. International Journal of Mineral Processing. 1983; 10(3): 183-204.

2 Uwadiale GGOO. Flotation of iron oxides and quartz: a review. Mineral Processing and Extractive Metallurgy Review. 1992;1 I:I29-161.

3 Al-Wakeel MI, El-Rahman MKA. Beneficiation of low grade Ghorabi iron ore, Bahariya Oasis, Egypt: a case study. Mineral Processing and Extractive Metallurgy. 2006; II5(4): I77-I82.

4 Ma M. The dispersive effect of sodium silicate on kaolinite particles in process water: implication for iron-ore processing. Clays and Clay Minerals. 201 I;59(3):233-239.

5 Zhang P, Snow R, Peres AEC, El-Shall H, El-Midany A. Depressants in nonsulfide minerals flotation. In: Fuerstenau MC, Jameson G, Yonn R, editors. Froth flotation: a century of innovation. Markham: SME; 2007. p. 555-574.

6 Marinakis KI, Shergold HL. Influence of sodium silicate addition on the adsorption of oleic acid by fluorite, calcite and barite. International Journal of Mineral Processing. 1985; 14:177- 193.

7 Qi GW, Klauber C, Warren LJ. Mechanism of action of sodium silicate in the flotation of apatite from hematite. International Journal of Mineral Processing. 1993;39:25I-273. 
8 Rao, S. R., Viajayakumar, T. V., Rao, S. S., Prabhakar, S. Raju, G. B. Effectiveness of sodium silicate as gangue depressants in iron ore slimes flotation. International Journal of Minerals, Metallurgy, and Materials. 20I I; I8(5):5I5522.

9 Arantes RS, Lima RMF. Influence of sodium silicate modulus on iron ore Flotation with sodium oleate. International Journal of Mineral Processing. 2013;125:157-160.

10 Lopes GM, Lima RMF. Flotação direta de minério de ferro com oleato de sódio. Rem: Revista Escola de Minas. 2009;62(3):323-329.

I I Nascimento DR, Pereira RD, Lima RMF. Influence of sodium silicate on floatability and charge of hematite and quartz with sodium oleate. Latin American Applied Research. 2013;43:189-191.

12 Potapova E, Yang X, Grahn M, Holmgren A, Forsmo SPE, Fredriksson A, et al. The effect of calcium ions, sodium silicate and surfactant on charge and wettability of magnetite. Colloids and Surfaces A: Physicochemical and Engineering Aspects. 20I I (I-3);386:79-86.

13 Jordean N, Marmier N, Lomenech C, Giffaut E, Ehrhardt J. Sorption of silicates on goethite, hematite and magnetite: experiments and modeling. Journal of Colloid and Interface Science. 2007;3 I2:224-229.

14 Jolstera R, Gunneriusson L, Forsling W. Adsorption and surface complex modeling on maghemite in aqueous suspensions. Journal of Colloid and Interface Science. 2010;342:493-498.

Recebido em: 03 Jun. 2016

Aceito em: 27 Ago. 2016 